\title{
Temporal Recurrence in Andrew Lewis's Penmon Point
}

\author{
Ambrose Seddon \\ Department of Creative Technology \\ Faculty of Science and Technology \\ Bournemouth University, UK \\ Email: aseddon@bournemouth.ac.uk
}

\begin{abstract}
In this chapter, Andrew Lewis's acousmatic composition Penmon Point will be analysed by considering the recurrent phenomena evident within the work. This approach aims to investigate the various webs of sound-material correspondences that develop and inform the listening experience as the music unfolds. A concept of recurrence pertinent to acousmatic musical contexts is briefly outlined in terms of (i) correspondences amongst sounds, and (ii) the different timescales over which those corresponding sounds recur. The significant types of sound material occurring within Penmon Point are then introduced, followed by an examination of their various sound qualities and source bonding, and the temporal relationships that their recurrences create. This analysis will focus on recurrences operating over more global timescales, and a variety of these higher-level, structural relationships are introduced and illustrated. Contrasting spatial perspectives and 'covert correspondences' are shown to be significant means through which recurrent phenomena are manifest, whilst structural patterning is considered in terms of 'sound-event chains'. The various observed aspects of recurrence are then contemplated with regard to what they bring to the interpretation of the work. In conclusion, the four-part question posed by this volume is addressed in relation to a recurrence-based analytical approach.
\end{abstract}

Keywords: music, acousmatic, recurrence, analysis 


\subsection{Introduction}

This analysis considers Andrew Lewis's acousmatic work Penmon Point (2002-

03) in terms of recurrent phenomena occurring over a number of different timescales. Investigating a work from this viewpoint involves considering the various kinds and types of sound materials present, assessing aspects of similarity or difference among them, noting how they function when they recur, and contemplating why they are significant. 'Before embarking on the analytical discussion, a brief clarification of the idea of recurrence, as it relates to acousmatic music, is necessary.

\subsection{Background to the adopted analytical approach}

Notions of recurrence extend beyond pure repetition, and hints, glimpses or vestiges of earlier material may be sufficient to remind the listener of preceding events, even though the original material has not reappeared explicitly. Such referrals among sound materials might be founded on different degrees of similarity, ranging from apparent sameness to just residual resemblance. Thus, recurrent phenomena might include returning states, types of sounds and sound sources, or derivations produced through transformation processes. Naturally, sound material must be lodged in memory to some degree for a recurrence to be perceived; it must be memorable. Furthermore, the sound material must be striking and differentiable from its surroundings in the first instance, and have a clearly discernible identity. If sound material appears among other similar sounds, or if it is masked in some way, its potential to make an impact may be reduced.

A recurrence-based approach to analysis concerns the ways in which sound materials correspond and might be related, particularly when they exhibit variability or only traces of earlier events. Correspondences among sound identities may be

- This approach was initially formulated with acousmatic works in mind (Seddon 2013), but can potentially be applied to many other genres such as (but not restricted to) instrument with electronic sound, IDM, or contemporary instrumental and vocal composition. 
perceived and usefully described in terms of shared spectromorphological ${ }^{2}$ attributes and/or shared source associations. Furthermore, corresponding sounds will invariably recur over different timescales, so the temporal relationships among them must be considered in order to better understand how recurrent phenomena operate at different levels of structure. Discrete sound events arranged over relatively short timescales may establish lower-level relationships, and these can be broadly thought of in terms of repetition and identity variation. These can often be considered to be operating at the gestural level. However, this analysis of Penmon Point will focus on the higher-level relationships at play i.e. the relationships among recurrent phenomena that provide a more global sense of structure and operating at the structural level. These may occur among discrete identities or events, as well as spatial environments or settings, ${ }^{+}$and are founded on the notion of return, implying that an earlier instance has been 'left behind' in some way.

The musical significance of recurrent sound material is also defined by the contextual role it fulfils when it returns, along with any expectations that it evokes based on the preceding instance(s). As such, the structural function of the recurrent material, and its degree of interaction with other sound materials present, must also be considered as these factors inform the observed temporal relationship. This analysis will draw upon the ideas of Smalley (1997) and Roy $(1996,1998,2003)$ in the

\footnotetext{
" "Spectromorphology is concerned with perceiving and thinking in terms of spectral energies and shapes in space, their behaviour, their motion and growth processes, and their relative functions in a musical context" (Smalley 1997, 124).

"The Oxford English Dictionary defines a "setting" as "the place or type of surroundings where something is positioned or where an event takes place," emphasising both configuration and location. In musical contexts, acousmatic images (a term used by Smalley (2007)) will appear both fleetingly as well as existing for more extended durations, so the concept of the setting as used here includes not just configuration and location, but a sense of temporal permanence and, in turn, the establishment of a spatial feel. It is not possible to define the setting in terms of a minimum duration, but rather its existence is rooted in a state of perceived permanence.
} 
description of structural functions and the behavioural relationships observed among the sound identities within a particular context. ${ }^{5}$

\subsection{Analysis}

Penmon Point (2002-03) features a variety of higher-level, relationships established by the recurrences of a limited number of sound types. These are often characterised by distinct source bondings ' and spectromorphological details, and can be summarised as:

- fast attack, slowly decaying inharmonic sounds, possessing bell-like source bondings to varying degrees;

- environmental seascapes, suggested by distant surf and bird cries;

- various proximate sounds, such as pebbles or stones rolling and colliding, wave-like noise-based morphologies, water trickling/flowing, snapping and breaking sounds;

- vocal utterances.

The following discussion will outline some of the different relationships at play, assessing their musical significance and how they contribute to the perceived structural organisation and the interpretation of the work as a whole.

\subsubsection{Bell identities}

Various bell sounds occur. These are all connected by the impression of a metallic, fast attack, slowly decaying resonance, whilst also sharing distinctive spectral characteristics based around a G\# pitch. Although the recurrences of these

\footnotetext{
shis analysis will also refer to concepts that have been developed as part of the author's doctoral research to help describe a variety of recurrence-based higher-level relationships. Terms have been selected to be self-explanatory.

- Higher-level relationship-types will appear in bold italics. Terminology referring to structural function and behavioural relationships will appear in italics.

"Smalley's concept of source bonding highlights "the natural tendency to relate sounds to supposed sources and causes, and to relate sounds to each other because they appear to have shared or associated origins" $(1997,110)$. Such bonding will influence the perception of a sound's identity and its musical significance.
} 
sounds might be grouped together because they are 'bell-like', forming a typological 'bell' grouping based upon that type of sound source, ${ }^{8}$ more specific sub-groups become apparent, largely defined by distinct source bondings. Indeed, the various bell types are distinguished by a number of sound qualities, such as: the impressions of space that they convey, their differing durations, and their more specific spectromorphological details.

The recurrent bell identities act as markers, particularly when the piece is considered as a whole (see Figure 1), delineating the unfolding sections. ${ }^{\circ}$ However, within the local contexts they function in further capacities.

\footnotetext{
- Correspondences among sounds can be established according to types of source and cause, resulting in 'typological' groupings and recurrences. This can be the case even if the individual sounds are perceived to be different, assuming that they are somehow similar in the kind or type of source-cause activity. A sound archetype founded on a particular sound source stimulates expectation and comparison based on the listener's real-world experience of that source. For example, an archetype described as 'wave-like' will consist of particular characteristics, which must be shared by the sounds that are part of the archetypal grouping.

'Markers are often similar sounds that draw attention to temporal position, marking sections or particular points within a work. Their recurrence jogs memory and creates a sense of temporal perspective, encouraging the appraisal of what has happened in between. Thus, markers might be considered recurrent musical landmarks.
} 


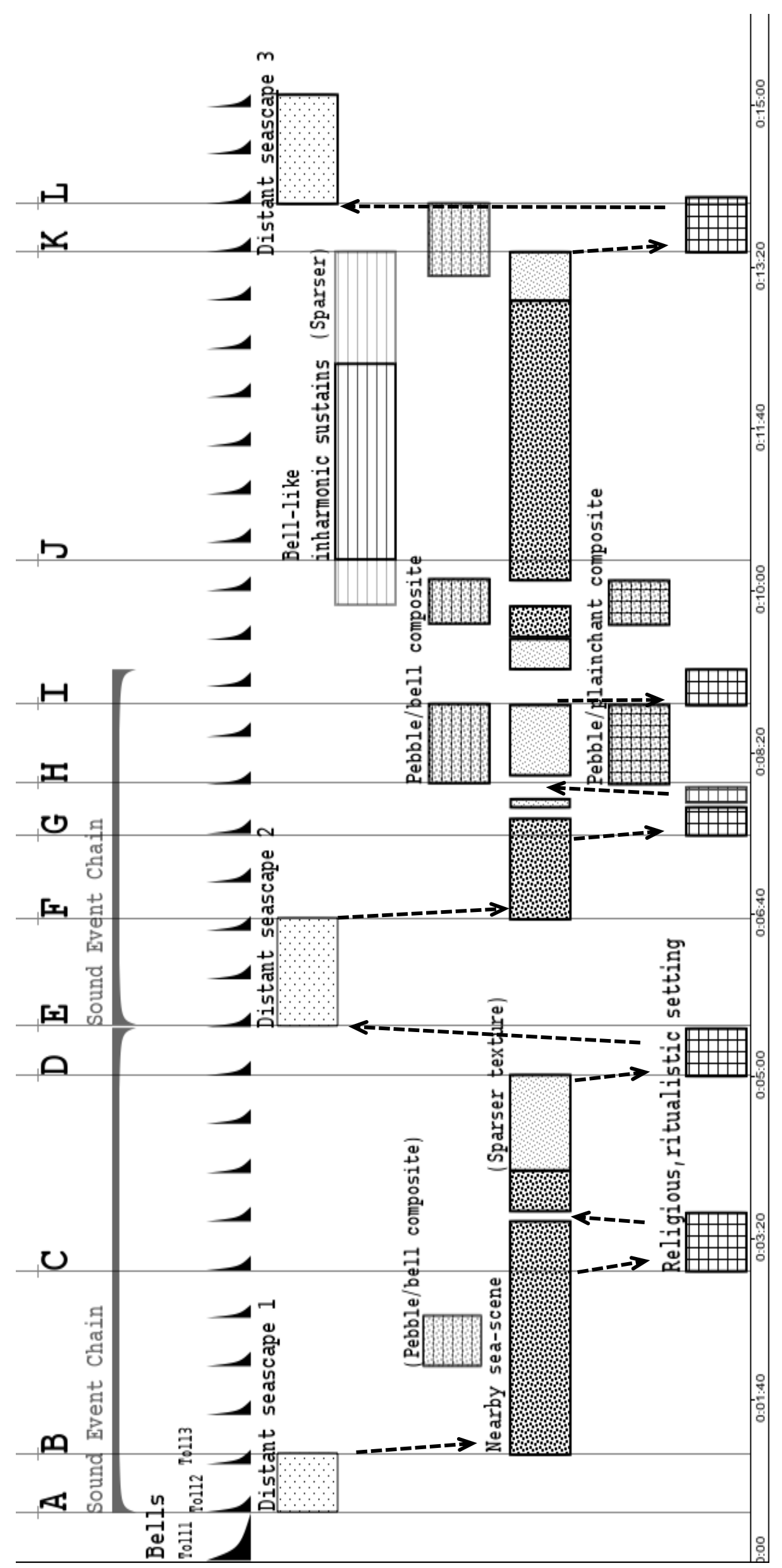

Figure 1: Overview of Lewis's Penmon Point 


\subsection{1.a. Illustrative bell recurrences}

\section{Toll $10^{\prime} 00$}

The spectromorphologically complex sound at the opening of the work commands attention much as a ringing bell might in everyday experience, and might be considered bell-like in broad terms. However, it exhibits some notable sound qualities. The extended resonance becomes partly absorbed by the additional layers of spectral swells and descending spectral contours, some emerging with accelerating and decelerating iterations. These subsequently withdraw, allowing the bell-like resonance to re-emerge and decay (audio ex. $\left.1,0^{\prime} 00-0^{\prime} 28\right) .{ }^{10}$ Due to the various layers, this sound might be described as a third-order surrogate (Smalley $1997,111-112)$, and its source-bonded spatiality is more remote from reality compared to many of the subsequent bells."

\section{Toll 2 0’30}

In contrast, the source-bonded lighthouse bell at 0 ' 30 is much shorter in duration, sounding distant due to the spectral restriction in both higher and lower frequency ranges (audio ex. 2, $0^{\prime} 28-0^{\prime} 38$ ). ${ }^{2}$ It is the second bell type, and as well as performing a marker function it signals the entry of a 'real-world' seascape environment, which coexists with the remaining continuant spectral residues of Toll 1 , resulting in two layered or superimposed spaces. It also reminds of Toll 1 since its

\footnotetext{
10 Audio resources for this chapter can be found at: http:// www.cambridge.org/9781107118324.
}

" "Third-order surrogacy is where a gesture is inferred or imagined in the music. The nature of the spectromorphology makes us unsure about the reality of either the source or the cause, or both. We may not be sure about how the sound was made to behave as it does, what the sounding material might be, or perhaps about the energy-motion trajectory involved" (Smalley 1997, 112).

${ }^{1}$ The subsequent presence of source-bonded seascape sound material reinforces this sense of space, which in turn strengthens the impression of this bell identity. The programme note accompanying the DVD-Audio reveals that the bell is actually that of Penmon Point lighthouse. 
fundamental pitch is the same. Contextual elements become significant; in retrospect, this bell instance is part of the newly introduced distant seascape setting due to the source-bonded spatial and contextual congruency between it and the seascape. ${ }^{\text {s }}$

\section{Toll 3 1'00}

The recurrence of the lighthouse bell at 1'00 reinforces that sound in our perception; it is embedded within the ongoing seascape environment and the sense of contextual congruency is strengthened (audio ex. 3, 0'54-1'04). Additionally, it creates a marker relationship (which extends as the piece unfolds) by delineating this local distant-seascape passage, and provoking the expectation that it could ring a third time. It also terminates the continuing spectral residues of toll 1, an action that connects the two superimposed spaces, yet does not instigate or signal any new material.

\subsection{1.b. Aspects of correspondence among subsequent bell tolls}

The subsequent bell instances exhibit different kinds of correspondence with tolls 1, 2 and 3. For example, the bell at 1'30 is spectrally brighter than tolls 2 and 3, and sounds to be more proximate (audio ex. 4, 1'28-1'34). The external lighthouse bell sound at 2'00 is synchronous with descending, pulsed, inharmonic spectral figurations reminiscent of toll 1 (audio ex. 5, 1'58-2'10). At 2'30, similar figurations coexist with a spectrally brighter and nearer bell toll, recalling that of 1'30 but appearing to be a composite of layered bell-like sounds (audio ex. 6, 2'27-2'51). And the instance at 3'00 features the lower-frequency spectral material of toll 1, simultaneous with the nearer bell-like material (audio ex. 7, 2'58-3'10). Thus the linear separation and recurrence of different elements of the initial bell sounds allows attention to focus on particular aspects of correspondence. It also stimulates a process of comparison between them and the subsequent instances, resulting in

: The idea of contextual congruence concerns whether sound material appears to be congruent with the current context, either in terms of its spectromorphology or source associations. 
networks of correspondence that establish some striking layered combinations based upon distinct sound qualities and the impressions of space evoked by the original tolls.

\subsection{1.c. Issues of function}

Following tolls 1, 2 and 3, the bell identities continue to fulfil the marker role, but they perform additional structural functions as indicated in Table 1 (audio ex. 8, $\left.0^{\prime} 00-5^{\prime} 50\right)$.

\begin{tabular}{|c|c|}
\hline Time & Functions attributed to bell recurrences \\
\hline 000 & - First occurrence \\
\hline $0 ’ 30$ & - Signal: signals entry of 'real-world' seascape \\
\hline I'00 & - Termination: terminates continuant spectral residues \\
\hline I'30 & - Interruption: briefly interrupts/halts the pebble-based texture, which then returns at I'35 \\
\hline 200 & - Termination: appears to terminate the proximate, pebble-based activity \\
\hline $2 ’ 30$ & $\begin{array}{l}\text { - Signal: synchronous with climax of pebble-based intensification/accumulation/release pattern at 2'28; } \\
\text { increases in motion, density and amplitude of the pebble-like granular material create a sense of } \\
\text { tension, which is released by the bell strike } \\
\text { - Specific context recurs at 7'00 }\end{array}$ \\
\hline $3 ’ 00$ & - Signal: signals spatial shift; ritualistic utterance space emerges out of the bell resonance \\
\hline $3 ’ 30$ & $\begin{array}{l}\text { - Instigation: instigates crescendo of the vocal-like graduated continuant, itself leading to the return of } \\
\text { the pebble/waves texture } \\
\text { - Termination: terminates the ritualistic utterance space }\end{array}$ \\
\hline $4{ }^{\prime} 00$ & $\begin{array}{l}\text { - Termination: terminates the pebble/waves activity (more quickly than at 2’00) } \\
\text { - Deflection: initiates change in textural emphasis }\end{array}$ \\
\hline 4'30 & - Instigation: instigates new continuant spectra (related to bell); instigates iterative descents \\
\hline $5 \cdot 00$ & $\begin{array}{l}\text { - Termination: terminates continuant bell-like spectral residues } \\
\text { - Instigation: instigates change to ritualistic utterance space }\end{array}$ \\
\hline $5 ’ 30$ & $\begin{array}{l}\text { - Termination: terminates ritualistic utterance space } \\
\text { - Instigation: instigates recurrence of environmental seascape }\end{array}$ \\
\hline
\end{tabular}

Table 1 Functions attributed to bell sounds in Penmon Point 0'00-5'50

An instigative function increasingly becomes associated with the bell sounds, and accordingly this passage conveys a considerable sense of forward motion. However, this impression changes at other points in the work. For example, between $10^{\prime} 00$ and 12 '30, the bell tolls largely coexist with the more slowly evolving sustain 
texture, wave and pebble sounds, and the sense of forward motion driven by the bell tolls is reduced; any expectations of instigative change are unfulfilled ( $\mathrm{J}^{\mathrm{w}}$, audio ex. 9, 9'58-12'30). Similarly, when the layered combination of the bell and seascape recurs at 5'30 (E), the bell acts as a sectional marker whilst also temporally dividing the seascape passage (at $6^{\prime} 00$ and reinforced at $6^{\prime} 30$ ). However, within this context the bell becomes established as an environmental feature, contributing to the evocation of the seascape (audio ex. 10, 5'25-6'48). When the tolls are embedded within the realistic sea setting, the notion of energetic forward motion is reduced.

Ritualistic, possibly religious, bells are the third distinct bell-type to occur (for example, at 5'15), and their source bonding conveys a particular internal spatiality (audio ex. 11, 5'12-5'34). ${ }^{15}$ They are higher in register compared to the work's opening bell-like sound and the lighthouse bells, yet they share the same pitch centre (approximately G\#), as is evident at 5’30 (E). At this point, the ritualistic bell fulfils the sectional marker function, terminates the sense of religious / ritualistic space (see 1.3.2.a below), and instigates textural and spatial change. ${ }^{16}$

Each bell recurrence reinforces our awareness of the bell archetype. Even the opening sound $\left(0^{\prime} 00\right)$ conforms to this archetype in broad terms (overall morphology and core spectral content), yet it might be best described as a 'hyper-bell'. It is belllike when compared to the following lighthouse tolls and ritualistic bell sounds, yet the extended resonance, expanded spectral range and additional spectral figurations suggest that it is highly transformed. Thus, the hyper-bell attains significance in retrospect due to the subsequent occurrence of more strongly source-bonded bells. (Of course, the recurrence of separate elements of the hyper-bell contributes to this

\footnotetext{
"Capital letters refer to locations indicated on Figure 1.

is Additional source-bonded sounds again reinforce the sense of space, in this case, plainchant utterances, bringing internal, ritualistic and religious associations.

"The coincidence of the ritualistic bell with the lighthouse bell underlines their pitch correspondence.
} 
impression.) This also suggests that groupings based upon sound-type_can occur retrospectively.

However, while the various bell sound identities manifest typological similarities, they convey contrasting cultural associations and meanings that establish sub-groups. For example, the ritualistic, ceremonial bell identities act as religious signifiers representative of cultural/spiritual ritual, while the external lighthouse bells could be associated with warning and danger. Of course, such interpretations are the products of culturally-learned contexts, and the degree of significance to the listener will depend on familiarity with the relevant cultural cues.

\subsection{1.d. Temporal distribution of bell recurrences}

One remains aware of the recurring bell tolls throughout, but they are sufficiently spaced in time not to be perceived as temporally regular (although they actually occur every 30 seconds, often delineating sections at the sequence level), and are largely perceived as sectional markers and landmarks. ${ }^{n}$ Impressions of regularity and temporal flow are influenced by the intervening sound materials and their source associations. Snyder suggests that the speed at which time is perceived to pass is related to familiarity with, and density of, sound events $(2000,213-215)$. In Penmon Point, the densely populated, active sections (for example, B, audio ex. 12, $1^{\prime} 05-2^{\prime} 10$, and audio ex. 13, 2'25-3'10) give the impression of time passing rapidly between the bell tolls. However, the calmer distant seascape setting (see 1.3.2 below) conveys continuity, regularity and a recognisably expansive sound source (the ocean), alluding to a sense of permanence and even stasis, particularly at 5'30-6'30 (E, audio ex. 10, 5'25-6'48). At this point the bell's marker role is less overt, and it fulfils an additional function by helping to maintain the setting and the section. This passage may also be perceived to take longer to unfold 'in the moment' because less seems to happen, and there is also a natural expectation for the setting to continue.

"The liner notes to the DVD-A indicate that the Penmon Point lighthouse bell actually tolls every 30 seconds. 


\subsubsection{Spatial perspectives}

The recurrence of source-bonded settings is a defining characteristic of Penmon Point, and the piece features three distinct setting-types.

\section{Distant seascape}

The distant seascape (initially appearing at A, 0'30) is a strongly sourcebonded, and largely unaltered, environmental phenomenon observed at distance (audio ex. 14, 0'28-1'15). The distant perspective is suggested by its restricted spectral resolution, and this setting conveys a sense of permanence both spectromorphologically (by remaining consistent and unaltered) and through the source bondings that it evokes— the sea is expected to continue making sound even when not heard.

\section{Nearby sea-scene}

The nearby sea-scene first appears at 1'10 (B), and features source-bonded sounds of: pebbles rolling and pounding together; wave-like noise structures; water trickling/flowing; snapping and breaking sounds (audio ex. 15, 1'05-1'59). These source associations in combination create a context suggesting a coastal location and experience. A sense of heightened activity, mobility and energy is conveyed, and these presumed details of the distant seascape suggest the energy of breaking waves on a pebble beach when close at hand. Both the distant seascape and the proximate sea-scene potentially allude to the same source (the sea experienced at a coastal location) but perceived from very different spatial perspectives. Accordingly, there is a generalised source association common among these sounds even though the specific sound sources themselves are actually different. ${ }^{\text {s }}$

\footnotetext{
"High- and low-register inharmonic sustains (presumably abstracted from the bell sound material) appear concurrently with the sea-scene sounds. This results in a mix of sound-types, spatial perspectives and locations, yet the spatially proximate, sea-related sounds remain the dominant feature.
} 


\section{Ritualistic/religious spaces}

The recordings of plainchant, first emerging at around 3'00 (C) but most clearly apparent at 5'00 (D, audio ex. 16, 4'59-5'32), bring with them impressions of human presence within a religious or ritualistic context. Reverberation reinforces the sense of an internal location, possibly suggesting a monastery or church, yet the concurrent internal resonance of the bell might also suggest that the voices are contained within the resonance. Potentially affective (depending on one's previous experience of plainchant), the recordings suggest a particular social and cultural scenario, conveying a contemplative, inward-looking emotional state.

\subsection{2.a. Recurrent settings}

The distant seascape, nearby sea-scene and ritualistic, religious space are encountered under different circumstances. Recurrent shifts and ruptures, as well as the recurrent settings in themselves, establish higher-level relationships that contribute to the structure of the piece. "The recurrent settings are indicated in Figure 1, with the shifts between them shown by arrows.

\section{Distant seascape}

Occurring at 0'30,5'30 and $14^{\prime} 00(\mathrm{~A}, \mathrm{E}$ and $\mathrm{L})$, the distant seascape delineates the higher-level structure by providing three setting-based, environmental markers or landmarks. The recurrences reinforce the seascape setting, and all of the instances feature similar onset functions, but with different durations and terminations.

Instigated by the lighthouse bell, each instance of the distant seascape contrasts strongly with the preceding material in spatial, spectral and source-bonded terms. The first seascape occurrence at 0'30 instils this setting in our perception, masking

\footnotetext{
1" Ruptures of, and shifts between, particular spaces, contexts and ongoing sounds can become significant recurrent features. Rupture implies suddenness, as if the existing impression is instantaneously shattered by a change to a new state or context, while shift involves a less sudden change, or a less overt contrast between what is shifted from and what is shifted to.
} 
(although not eradicating) the spectral and spatial impression of the opening 'hyper bell' (audio ex. 17, 0'00-0'42). This masking is striking and creates a partial rupture, with the seascape now the dominant element within the music. The two recurrences (5'30 (E) and 14'00 (L)) categorically rupture ritualistic, religious settings, the rupture process enhancing the impact of the seascape on both occasions and establishing a distinct spatiomorphological trajectory (audio ex. 18, 5'25-5'40, and audio ex. 19, 13'50-14'15). ${ }^{20}$ These later ruptures feature changes from pitch-based to noise-based texture, intimacy to remoteness, internal to external space, and social/cultural human presence to solitude in an outside environment. Accordingly, the listening perspective changes from intimate engagement to more remote observation, resulting in recurrent processes of withdrawal in spatial terms, and termination of the preceding setting/section in functional terms.

The distant seascapes are longer in duration when they recur, spanning three of the regular bell tolls instead of two. This creates an increased sense of stability and permanence from 5'30 (E) while also thwarting the expectation of an imminent setting shift. The sense of permanence is reinforced at $14^{\prime} 00(\mathrm{~L})$. However, the methods of termination change. The distal seascapes at 0'30 (A) and 5'30 (E) are terminated by shifts to the next sea-scene (audio ex. 14, 0'28-1'15, and audio ex. 10, 5'25-6'48). This reinforces a spatiomorphological process that results in increased energy, mobility, proximate spatial focus, and a sense of forward motion. The music 'zooms in' to the details of the seascape, offering a new, immersive perspective on the distantly observed, source-bonded phenomenon. However, the final distant seascape $\left(14^{\prime} 00-15^{\prime} 00, \mathrm{~L}\right)$ is terminated by the third lighthouse toll, whose elongation and gradually dissipating energy, along with the subsequent bass sound, conveys a sense of closure. This subverts any expectation of a return to the proximate sea scene (audio ex. 20,13'50-15'25). Thus, while the three distant seascape sections sound

${ }^{2}$ The sense of the ritualistic space is enhanced on both occasions by the high-pitched bells, which stimulate further religious connotations. In retrospect, these bells function by anticipating and marking the terminating rupture and change to the distal seascape, and this recurrent process suggests that the music is nearing the end of a larger scale section. 
similar and evoke common source bondings, they feature changing structural functions, fulfilling different roles within the music.

The distant seascape attains particular significance when it recurs. The instance at 5'30 (E) becomes a notable point of energetic calm and stability relative to the more active preceding sections. Additionally, it helps define the spatial bounds of the piece so far by returning to the earlier distant location, instilling a sense of closure to the sequence of contrasting settings. These musical attributes are not apparent until the seascape recurs; they are largely determined by the nature of the intervening sound material, and the significance of the seascape is delayed until this point.

\section{Ritualistic settings}

Recurrences of the religious, ritualistic setting are also the result of shift and rupture. It initially emerges at 3'00-3'30 (C) amid nearby wave sounds, yet the potential shift to this new space is not completed, the music soon returning to the nearby sea-scene (audio ex. 21, 2'55-3'45). However, this initial instance anticipates the more explicit recurrence at 5'00 (D), which reinforces and elaborates the setting through clearer human utterances and human-caused bell strikes. ${ }^{21}$ Instigated by a bell toll, this instance of the religious, ritualistic setting definitively ruptures the sparser preceding bell-like spectral residues and pebble-like gestures to become the dominant element, emphasising its musical significance (audio ex. 22, 4'50-5'35). ${ }^{2}$ Furthermore, although less marked than the earlier ruptures (A and B), the recurrent

\footnotetext{
${ }^{2}$ These two instances might also be considered as an announcement/reminder pair, in Roy's sense. "An announcement states a fragment of the reminder in order to prepare for, and to increase the perceptual importance of, the complete event that will appear later. The announcement and the reminder may be separated by a long time span; an announcement can have many reminders but a reminder has only one announcement" $(1998,113)$.

${ }^{2}$ The bell at $5^{\prime} 00$ could now be seen to possess religious connotations (a church or monastery bell) due to the context suggested by the plainchant. Its decay becomes part of the ensuing setting.
} 
rupture function is now conveyed by different sound materials, suggesting structural functions can noticeably recur even if the sounds themselves are different. ${ }^{2 s}$

\subsection{2.b. Sound-event chains}

The pattern of settings and transitions from $0^{\prime} 30-5^{\prime} 30$ largely recurs in a similar order from $6^{\prime} 30-9^{\prime} 00$, forming a recurrent, setting-based sound-event chain but of shorter duration. ${ }^{24}$ Recurrences of both the settings, their sequential arrangement, and the shift/rupture types remind of the earlier instances, reinforcing them while featuring local variations, changes in gestural pacing and elaborations. Indeed, additional material is featured at $8^{\prime} 00-8^{\prime} 30$.

However, at 9'10, instead of returning to the distant seascape after the plainchant recurrence (as occurred at 5'30), the music moves, via the sparser proximate sea-scene, to a new section ( $\left.\mathrm{J}, 10^{\prime} 20-12^{\prime} 30\right)$ dominated by sustaining sounds that correspond spectrally with the bell (audio ex. 9, 9'58-12'30). Any expectation of a return to the distant seascape is unfulfilled. The eventual recession of the bell-related sustains allows attention to focus on the sparser proximate sea-scene. The subsequent shift from the sea-scene to the returning religious, ritualistic space at 13'30 (K; now existing alongside a low amplitude, 'pebble/bell' composite sound, discussed below) reinforces this particular setting transition whilst creating an impression of partial closure. The music is back in familiar territory following the more 'abstracted' setting. Significantly, the ruptural return of the wave sounds at $14^{\prime} 00$ (L) completes the closure sequence, with the sense of sectional termination largely conditioned by the similar (and similarly closural) spatial movement at 5’30. Additionally, the waves and distant seascape at $14^{\prime} 00$ (L) seem nearer than in the earlier distant seascapes (increased high-frequency resolution and greater definition

\footnotetext{
${ }^{2}$ Later recurrences of the ritualistic, religious setting exhibit similar emergent and ruptural structural functions.

${ }^{2}$ Recurrent linear arrangements of sounds can establish sound-event sequences, or chains. The identity of such a chain will be determined by the sounds present, their sequential occurrence or order, their structural function and the nature of the temporal relationships among them.
} 
in the wave sounds) yet the appearance on its own, and the low degree of volatility, reminds of the distant seascape rather than the more energetic nearby sea scene.

\subsubsection{Covert correspondences}

Covert correspondences, in terms of specific sound qualities, exist among certain sustaining sounds and the bell spectra, connecting apparently novel material to identities that are central to the work. For example, the proximate sea-scene at 1'10-2'00 (B) features inharmonic sustains that create a spectral covert correspondence (of fixed pitch centre) with the bell sound heard at the end of the passage (audio ex. 12, 1'05-2'10). The texture of inharmonic sustains at 10'20-12'30 (J) exhibits a similar covert correspondence; the metallic, inharmonic sound quality suggests a generic spectral connection to the bell sounds, which are the other significant 'metallic' materials of the piece (audio ex. 9, 9'58-12'30).

Some identities exhibit multiple covert correspondences. ${ }^{.5}$ A recurrence of the plainchant at 7'30 (G) appears over two simultaneous pitch-centres (audio ex. 23, 7'27-7'45). The higher pitch-centre recalls the plainchant material of 5'00 (itself spectrally corresponding with the recurring bell sounds), while the lower instance sounds more 'naturally' vocal and untransformed. A similar spectral duality occurs in the subsequent passage from $8^{\prime} 00-8^{\prime} 50(\mathrm{H})$. This features pebble-like sounds exhibiting bell-like and vocal-like spectra, forming 'pebble/bell' and 'pebble/vocal' composites that enmesh the two 'parent' sounds' identities. (The original pebble-like sounds can be heard in audio ex. 24, 1'33-1'44). ${ }^{26}$ The 'pebble/bell' and 'pebble/vocal' composites thus feature multiple covert correspondences with various parent identities. While their spectra are not exactly the same as the bell and plainchant material, the sense of correspondence is palpable, in effect blending

\footnotetext{
${ }_{25}$ These composites remind of a similar type of identity at 2'00-2'30.

${ }_{26}$ The pebble-like spectromorphologies appear in this extract amid inharmonic graduated continuants and breaking/snapping sounds.
} 
together or enmeshing the contrasting parent sound materials (for example at $8^{\prime} 01-$ $8^{\prime} 05,8^{\prime} 11-8^{\prime} 16$, and $8^{\prime} 25-8^{\prime} 32$, all passages heard in audio ex. 25). ${ }^{27}$

These composites privilege particular sound qualities while remaining rooted in the existing sound world; they remind of the bell-like spectra and the pebble-like activity, yet move away from the parent sounds by defining their own identity and spatiality. Of course, such conclusions are conditional on hearing the parent material already.

\subsubsection{Reflection}

Lewis's Penmon Point is a compelling evocation of place conveyed by a finely balanced musical structure. The recurrent sounds operate to present and reinforce the different aspects of that location, providing a clear impression of its fundamental characteristics. Higher-level relationships are formed throughout the work in a variety of ways, and returning impressions of space significantly contribute to the evolving sense of form.

The recurring bells delineate the work, marking section boundaries and sectional progression. Although regular in 'clock time' (as is the actual bell of the Penmon Point lighthouse), the bells' precise division of time is not immediately apparent. Lewis manipulates the listener's different experiences of time through the various kinds of intervening sound materials, be they the close-up, energetic sea scene, the distant seascape, the 'indoors' religious / ritualistic settings, or the more abstracted bell-like sustains. Each of these conveys a specific notion of time unfolding, and, in sequence, results in a sense of ebb and flow that affects the

\footnotetext{
${ }^{2}$ Identities featuring pebble-like morphologies and resonant-pitch spectra are heard earlier at $2^{\prime} 00-2$ '30, yet they do not spectrally correspond with the bell or vocal material. However, these identities do correlate with the 'pebble/bell' and pebble/vocal' composites in broad terms due to their similar morphology and generally pitch-focused spectral character, potentially preparing the listener for these kinds of sound materials.

$\approx$ These composites recur again at 13'30, but with more stable morphological activity and spectral content, and the reduced energy instils a sense of imminent closure.
} 
temporal experience of the work as a whole. ${ }^{2}$ Rather than experiencing rigid '30second' blocks, one encounters a variety of rates of activity, which shape our experience of time.

The idea of 'the bell' provides a fundamental unifying strand in the music, reflecting a sound that occurs in, and defines, the actual location of Penmon Point. Although the bell sound-type accounts for a number of distinct sound identities, the notion of 'bellness' is the central element around which the various environments of the composition are situated. The different kinds of bells complement the various sea-related sounds and vocal material to evoke coastal environmental and ritualistic/religious associations, resulting in a very specific sense of location. In a sense, the various bells together create a metaphorical vantage point from which the different scenarios are encountered; the bell is never 'far away', and each passage is experienced with reference to a bell of some sort.

Over longer timescales the distant seascapes delineate a two-part structure in which materials and settings in the first section are revisited, reinforced and elaborated in the second. Accordingly, the form evolves in a loosely cyclical way based upon the recurrent chain of sound events and settings, yet elaborations are explored in the latter stages of the second cycle. The significance of these later passages depends on the earlier prevalence and reinforcement of source-bonded sounds, the sound world becoming perceivably 'abstracted'. Indeed, an important feature of the work is that sounds that are potentially evocative of different lived, bodily experiences (i.e. 'I can see/hear the distant sea; I am near/in the surf; I am in a church or monastery') are the basis of the more abstracted passages. Lewis enmeshes many of the sounds (pebbles, bell resonances, voices) to create new hybrids, fusing specific sound qualities and source associations, and stimulating the reappraisal of the preceding material. The covert correspondences (including the fused identities,

\footnotetext{
${ }^{*}$ It is acknowledged that while the diagrammatic representation is useful in discussing or pinpointing certain features, it is more of an architectural overview, and cannot be considered a true representation of temporal experience.
} 
but also the bell-like spectral sustains) create correspondences with remembered scenarios, but also define new perspectives on them. From a formal perspective, the abstracted sounds provide considerable elaborations within the sound world, taking the music to new territories, yet paving the way for the eventual return of more familiar material. They are sufficiently remote from the original sounds to make the final recurrences of the nearby sea-scene, religious setting and distant seascape notable, creating a sense of wholeness and resolution by returning to those familiar scenarios. Perhaps the listener attends more fully to those corresponding qualities in the earlier sounds when hearing the work again, being more 'tuned in' to those aspects of the sounds' identities. Furthermore, the abstracted sounds are poetically significant, existing in and defining new spaces, intimately mixing and blurring the boundaries between the 'parents' to create an environment derived from the originally distinct sources: bells, sea sounds and vocal utterances. In a sense, these sounds embody a particular view of what Penmon Point is-the confluence of cultural/ ritualistic spaces, human presence and environmental phenomena.

\subsubsection{Closing remarks}

In considering what we want from the analysis of electroacoustic music and how might we get it, this book proposes a four-part question: Which tools / approaches? For which works / genres? For which users? With what intentions?

Regarding tools/approaches, this analysis has illustrated some of the ways in which recurrent phenomena can be considered within an acousmatic composition. Whilst the underlying concepts have been developed through the consideration of acousmatic music more generally, they can potentially be applied to other genres including (but not restricted to) instrument(s) with electronics, IDM, or contemporary instrumental and vocal composition. Indeed, the ideas of recurrence as demonstrated here may provide useful approaches to the analysis of works in 
which a solid pitch language is not clearly evident, and works that depend on other criteria for form to cohere.

Considering which users, whilst it is hoped that music analysts will find the concepts illuminating and usable, these ideas can be adopted in less-formal, real-time listening contexts, for example, concert performances and private listening. In this sense, the consideration of recurrent phenomena provides a listening strategy, which can help to articulate and to make sense of particular aspects of listening experience. Furthermore, the ideas are equally valuable to composers, potentially stimulating new approaches to sound organisation and transformation in terms of correspondences among sounds, and the deployment of those materials over different timescales.

What is the intention of such an analysis? What might be achieved? By adopting a recurrence-based listening strategy, listeners, analysts and composers are engaged with the sound materials from which the work has been composed and how these have been organised. This approach heightens awareness of the sounds present, their distinctive sound qualities and aspects of correspondence, and the temporal relationships at play. Whether considering covert correspondences or more overt recurrences, sensitivity to recurrent phenomena offers an essential way to consider and understand this fundamental aspect of musical organisation. 


\section{References}

Lewis, A. 'Penmon Point'. 2002-03. On Miroirs obscurs. Empreintes Digitales. IMED 0789 (2007). DVD-Audio.

Roy, S., 1996. Form and referential citation in a work by Francis Dhomont. Organised Sound, 1 (1), 29-41.

Roy, S., 1998. Functional and implicative analysis of Ombres Blanches. Journal of New Music Research, 27 (1-2), 165-184.

Roy, S., 2003. L'analyse des musiques électroacoustiques: Modèles et propositions. Paris: L'Harmattan.

Smalley, D., 1997. Spectromorphology: explaining sound-shapes. Organised Sound, 2 (2), 107-126.

Smalley, D., 2007. Space-form and the acousmatic image. Organised Sound, 12 (1), 3558. 